\title{
Antiapoptotic effect of found in inflammatory zone (FIZZ) I on mouse lung fibroblasts
}

\author{
MJ Chung, T Liu, M Ullenbruch and SH Phan* \\ Department of Pathology, University of Michigan Medical School, Ann Arbor, MI, USA
}

* Correspondence to:

SH Phan, Department of Pathology, University of Michigan

Medical School, Ann Arbor, Ml

48109-2200, USA.

E-mail:sphan@umich.edu

No conflicts of interest were declared.

Received: 5 September 2006 Revised: 5 February 2007 Accepted: 13 February 2007

\begin{abstract}
Myofibroblasts play an essential role in the abnormal deposition of extracellular matrix in pulmonary fibrosis. The presence or prolonged survival of these cells may be a key factor in the pathogenesis of progressive pulmonary fibrosis. Found in inflammatory zone (FIZZ)1 can induce myofibroblast differentiation and has an antiapoptotic effect on embryonic lung explant cultures. In this study, we investigated whether FIZZ1 also has an antiapoptotic effect on mouse lung fibroblasts (MLFs). Cells were treated with FIZZ1 for $24 \mathrm{~h}$ and then apoptosis was induced by TNF $\alpha$ in the presence of cycloheximide (CHX). FIZZ1 exhibited an antiapoptotic effect in MLFs, as assessed by flow cytometric analysis and TUNEL staining. Moreover, the cell number was higher in the FIZZ1-treated group relative to the nontreated control group after treatment with TNF $\alpha$ and CHX. FIZZ1 treatment also inhibited the apoptotic agent-induced activities of caspase-3 and caspase-8. Examination of potential signalling pathways revealed that FIZZ1 induced rapid phosphorylation of ERK-1/2, while PD98059, a MEK/ERK inhibitor, markedly induced activation of caspase-3. This antiapoptotic effect of FIZZ1 was associated with induction of myofibroblast differentiation in response to FIZZ1 stimulation. Taken together, these findings suggest that FIZZ1 is involved in pulmonary fibrosis through both induction of myofibroblast differentiation and increased or prolonged survival of myofibroblasts. This effect of FIZZ1 was mediated by inhibition of caspase-3 and -8, with involvement of the ERK pathway.

Copyright (c) 2007 Pathological Society of Great Britain and Ireland. Published by John Wiley \& Sons, Ltd.
\end{abstract}

Keywords: FIZZ1; myofibroblast; apoptosis; caspase-3; caspase-8; ERK

\section{Introduction}

FIZZ1 (found in inflammatory zone 1), also known as RELM- $\alpha$ (resistin-like molecule $\alpha$ ), was originally discovered in lung allergic inflammation [1], but its role is unclear. The FIZZ/RELM family has four members, FIZZ1/RELM- $\alpha$, FIZZ2/RELM- $\beta$, FIZZ3/resistin, and RELM- $\gamma$, characterized by their conserved 10-cysteine residue motif, with two members, resistin and FIZZ2, having an additional cysteine residue [1]. FIZZ1 is a $9.4 \mathrm{kDa}$ secreted protein and is expressed in white adipose tissue, spleen, lung, heart and mammary glands [1-3]. FIZZ1 inhibits adipocyte differentiation and nerve growth factor-mediated dorsal root ganglion survival. In normal lung, FIZZ1 expression is essentially undetectable, but in allergic airway inflammation it is highly expressed by macrophages, bronchial epithelium and type II alveolar epithelial cells (AECs) [1]. FIZZ1 is also highly induced in bleomycin-induced lung fibrosis and can induce myofibroblast differentiation in lung fibroblast culture [4]. The Th-2 cytokines, IL-4 and IL-13, induce AEC FIZZ1 expression via STAT6 and JAK-1 [5]. STAT6, or IL-4 and/or IL-13 deficiency abolish lung FIZZ1 expression and fibrosis in the bleomycin model. FIZZ1 has the capacity to promote lung cell proliferation, angiogenesis and inflammation [6,7]. Its expression is an indicator of alternative activation of macrophages [8,9]. All of these properties indicate a role for FIZZ1 in the regulation of cell growth/proliferation and differentiation.

During active fibrosis, de novo genesis of the myofibroblasts is a key common feature and it is thought that, due to their importance as a major cellular source of extracellular matrix and fibrogenic cytokines, their persistence or survival may contribute to progressive fibrosis instead of resolution and complete healing. Thus, understanding the basis for their survival may provide further insight into their role in the pathogenesis of progressive pulmonary fibrosis. Germane to this issue is the previous observation that, in addition to myofibroblast differentiation, FIZZ1 has antiapoptotic effects on embryonic lung explants and causes increased lung cell density [10], consistent with its mitogenic effect on pulmonary vascular smooth muscle cells [6]. However, the effect of FIZZ1 on lung fibroblast or myofibroblast survival/apoptosis and proliferation is unknown. 
In this study we hypothesized that FIZZ1 may have antiapoptotic or proliferative effects on lung fibroblasts, which can impact on the survival or persistence of the myofibroblast in the context of pulmonary fibrosis. We examined the antiapoptotic role of FIZZ1 in cultured mouse lung fibroblasts (MLFs), using biochemical assays, flow cytometric analysis and TUNEL staining, and surveyed potential associated signalling pathways. The results showed that FIZZ1 had protective effects against apoptosis in MLFs, which were mediated by the ERK signalling pathway.

\section{Materials and methods}

\section{Cell culture}

Six-week old female CBA/J mice were purchased from the Jackson Laboratory (Bar Harbor, ME). All animal experiments were carried out in accordance with the guidelines of the University Committee on the Use and Care of Animals (UCUCA) of the University of Michigan for the care and use of laboratory animals and with the Committee's approval. Lung fibroblasts were isolated as before [11] and cultured in DMEM, supplemented with $10 \%$ plasma-derived serum (PDS; Cocalico Biologicals Inc., Reamstown, PA), $100 \mathrm{U} / \mathrm{ml}$ penicillin, $100 \mu \mathrm{g} / \mathrm{ml}$ streptomycin, $0.25 \mu \mathrm{g} / \mathrm{ml}$ fungizone, $1 \%$ ITS (insulin, transferrin, selenium; Sigma, St. Louis, MO), $5 \mathrm{ng} / \mathrm{ml}$ PDGF (R\&D Systems, Minneapolis, MN) and $10 \mathrm{ng} / \mathrm{ml}$ EGF (R\&D Systems). The cells were used at passages 3-5.

\section{Induction and detection of apoptosis}

Cells $\left(5 \times 10^{5}\right)$ were plated onto $60 \mathrm{~mm}$ plates and when subconfluent they were serum deprived in DMEM with $0.5 \%$ PDS for $24 \mathrm{~h}$. Apoptosis was induced by treatment with $5 \mathrm{ng} / \mathrm{ml} \mathrm{TNF} \alpha$ (R\&D Systems) and $500 \mathrm{ng} / \mathrm{ml}$ cycloheximide (CHX) for $4 \mathrm{~h}$, or $200 \mathrm{ng} / \mathrm{ml}$ anti-Fas monoclonal antibody (BD, San Diego, CA) and $5 \mu \mathrm{g} / \mathrm{ml}$ CHX for 14-16 h. Since FIZZ1 induces myofibroblast differentiation after $24 \mathrm{~h}$ treatment in lung fibroblast culture [4,5], the antiapoptotic effect of FIZZ1 was studied by pretreating cells with $25 \mathrm{ng} / \mathrm{ml}$ murine FIZZ1 (Leinco Technologies, St. Louis, MO) for $24 \mathrm{~h}$ before $\mathrm{TNF} \alpha / \mathrm{CHX}$ treatment. Apoptosis was evaluated using annexin $\mathrm{V}$-propidium iodide (PI) staining (TACS ${ }^{\mathrm{TM}}$, Annexin V-FITC; R\&D Systems). Briefly, cells were collected after trypsinization, washed with ice-cold PBS and resuspended in $100 \mu \mathrm{l}$ Annexin V incubation reagent $(10 \mu \mathrm{l} 10 \times$ binding buffer, $10 \mu \mathrm{l}$ propidium iodide, $1 \mu \mathrm{l}$ Annexin V-FITC, $79 \mu \mathrm{l}$ distilled water) at a concentration of $3-5 \times 10^{5}$ cells $/ 100 \mu \mathrm{l}$, and incubated for $15 \mathrm{~min}$ at room temperature (RT) in the dark. Samples were washed with binding buffer and analysed by FACS Caliber (Becton-Dickinson). Apoptotic cells were identified as an annexin-V-positive/PI negative population.
Additionally, TUNEL assay was performed for in situ detection of apoptotic cells, using the ApopTag Plus Fluorescein In Situ Apoptosis Detection Kit (Chemicon, CA, USA). Induction of apoptosis and FIZZ1 treatment were identical to the method used in FACS analysis of apoptosis. This was followed by determination of apoptotic cells, in accordance with the manufacturer's protocol, and fluorescence microscopic analysis by counting the number of positive cells in randomly chosen, non-contiguous, high-power ( $\times 40$ objective) fields until a minimum of 400 total cells were counted. Cell number was counted after $48 \mathrm{~h}$ starvation with or without FIZZ1 treatment, using a Coulter counter.

\section{Cell transfection}

A dominant negative Akt construct was used to evaluate the role of Akt in response to FIZZ1 treatment. The construct, Akt-K179A, had a point mutation with a substitution of alanine for lysine at position 179 (gift from Dr Victor Thannickal, University of Michigan). The empty vector PCIS2 was used as negative control. Transfection of these constructs by Nucleofector technology was performed according to the optimized protocols provided by the manufacturer (Amaxa Biosystem, Cologne, Germany). Briefly, the cells were gently resuspended in $100 \mu$ primary mammalian fibroblast nucleofector solution (Amaxa Biosystem), mixed with cDNA and pulsed with the program U-23. Immediately afterwards, the cells were transferred into prewarmed fresh medium in six-well plates. The cells were used $24 \mathrm{~h}$ after transfection to evaluate the involvement of Akt in the antiapoptotic effect of FIZZ1. A control vector, pmaxGFP (Amaxa Biosystem), was used to gauge transfection efficiency.

\section{Western blot analysis}

Whole cell lysates were prepared and analysed by western blotting for the protein species indicated, as previously described [4]. The following antibodies were used: anti-Akt, anti-phosphoAkt and anti-ERK1/ ERK2 (R\&D systems); anti-phosphoERK1/ERK2 (Thr 202/Tyr204) (Cell Signalling, Beverly MA); anti$\alpha$-smooth muscle actin and anti-caspase-3 (Sigma, St. Louis, MI); and anti-caspase-8 (BD, San Diego, CA). Blots were developed using corresponding HRPconjugated secondary antibodies, followed by chemiluminescence detection (New England Biolabs, Beverly, MA).

\section{Caspase- 3 activity assay}

The bioactivity of caspase- 3 was measured with a Colorimetric Activity Assays Kit (Chemicon International, Temecula, CA). In brief, MLFs were resuspended in lysis buffer and centrifuged. The supernatants were incubated with caspase-3 substrate AcDEVD-pNA. The free pNA from Ac-DEVD-pNA was measured at $405 \mathrm{~nm}$, using a microplate reader. 


\section{Results}

\section{Antiapoptotic effect of FIZZI on mouse lung fibroblasts}

In preliminary observations, after serum and growth factor deprivation for $48 \mathrm{~h}, 5.5 \pm 0.29 \%$ (mean $\pm \mathrm{SE}$, $n=4$ ) of non-treated MLF underwent early apoptosis, as evaluated by annexin V-PI staining with analysis by flow cytometry. In cells treated with FIZZ1 $(25 \mathrm{ng} / \mathrm{ml})$, the number of apoptotic cells was significantly $(p<0.05)$ reduced to $3.7 \pm 0.54 \%$ (mean \pm SE, $n=4$ ) of total cells. This suggested the antiapoptotic activity of FIZZ1 in lung fibroblasts, which correlated with its myofibroblast differentiationinducing activity [4]. However, due to the relatively low level of apoptosis induced by serum deprivation, another approach is required to optimally study this effect. Optimal conditions for inducing apoptosis were observed when cells were treated for $4 \mathrm{~h}$ with $5 \mathrm{ng} / \mathrm{ml} \mathrm{TNF} \alpha$ and $500 \mathrm{ng} / \mathrm{ml} \mathrm{CHX}$, or for $14-16 \mathrm{~h}$ with $200 \mathrm{ng} / \mathrm{ml}$ anti-Fas and $5 \mu \mathrm{g} / \mathrm{ml}$ CHX. $\mathrm{TNF} \alpha$ or Fas alone had no effect on apoptosis under these conditions. TNF $\alpha / \mathrm{CHX}$ treatment caused a fourfold increase (to $22.5 \pm 0.46 \%$ ) in apoptotic cells by annexin V staining (Figure 1A, B). Pretreatment with $25 \mathrm{ng} / \mathrm{ml}$ FIZZ1 significantly $(p<0.05)$ diminished TNF $\alpha / C H X$-induced apoptosis by $>40 \%$. Anti-Fas antibody/CHX treatment showed a greater proportion of apoptotic cells $(26.1 \pm 0.79 \%)$, which was also significantly $(p<0.05)$ reduced by $32 \pm 4 \%$ by FIZZ1 pretreatment.

To confirm that FIZZ1 has an antiapoptotic effect, TUNEL staining was used as a complementary approach. The results confirmed that FIZZ1 had an antiapoptotic effect on lung fibroblasts (Figure 1D, E). FIZZ1 did not affect the basal or control level of apoptosis. Thus, using three different approaches to monitor apoptosis, FIZZ1 was found to have significant antiapoptotic activity. (a)
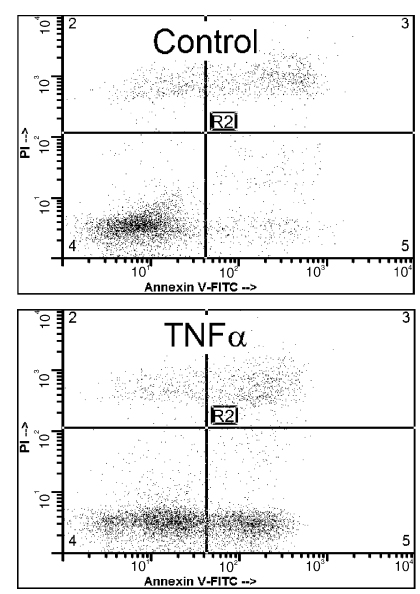

(c)
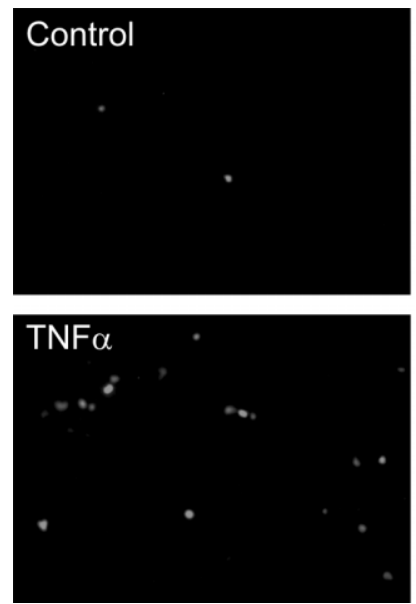
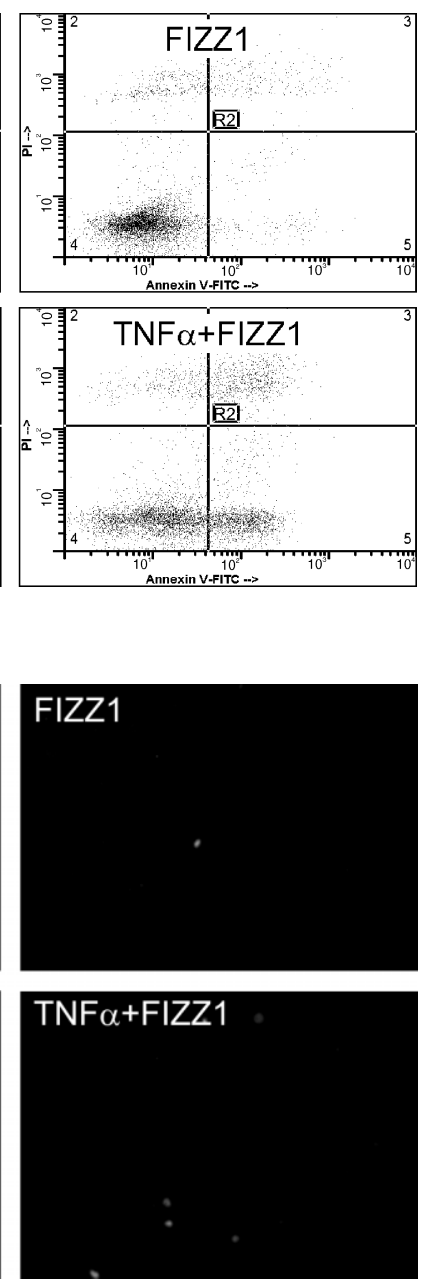

(b)

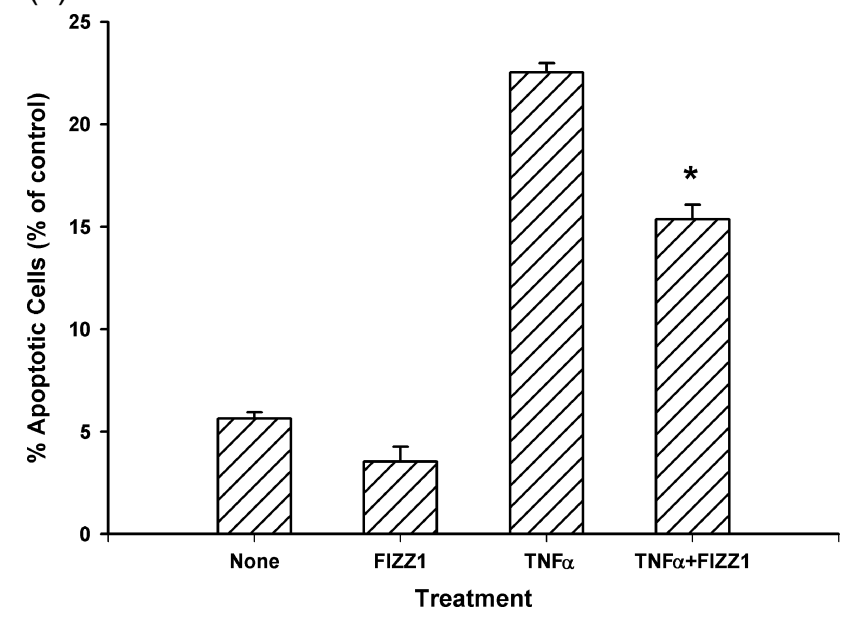

(d)

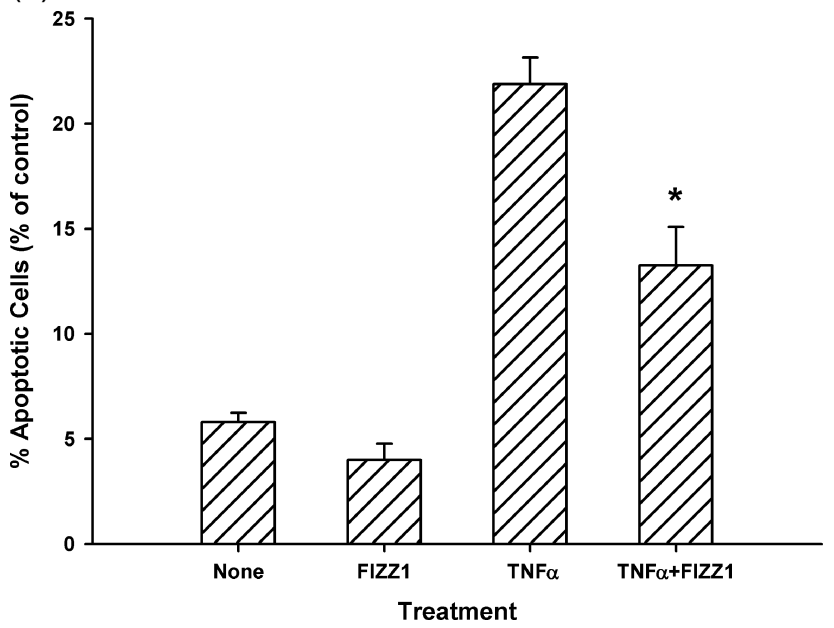

Figure I. Effect of FIZZI on mouse lung fibroblast (MLF) apoptosis. Apoptosis in MLF was detected by FACS analysis after annexin $\mathrm{V}$-propidium iodide (PI) staining (A, B) and by TUNEL assay (C, D). Cells were cultured in the absence or presence of FIZZI for $24 \mathrm{~h}$ and then treated with TNF $\alpha / \mathrm{CHX}$ for $4 \mathrm{~h}$. Representative FACS histogram (A) and micrograph from TUNEL assay (C) are shown. The experiments were repeated twice and the combined results from the respective assays are shown (B, D) as mean $\pm S E(n=3)$. In $(B, D)$, an asterisk indicates that the mean value for the TNF $\alpha+F I Z Z I$ group was significantly different $(p<0.05)$ from that for cells treated with TNF $\alpha / \mathrm{CHX}$ ('TNF $\alpha$ ') only, as assessed by ANOVA 


\section{Effects of FIZZI on caspase- 3 and caspase-8}

Since the death signal of $\mathrm{TNF} \alpha$ is mediated by caspase- 8 activation, and caspase- 3 is one of its downstream targets, the importance of these caspases to the antiapoptotic activity of FIZZ1 was examined. The TNF $\alpha$-induced activation of these caspases was confirmed by examining the cleavage of procaspase- 3 and -8 by western blotting. Cleavage of caspase- 3 to its $17 \mathrm{kDa}$ form was readily observed at $4 \mathrm{~h}$ after TNF $\alpha / \mathrm{CHX}$ treatment by western blotting, while caspase- 8 activation was detected as early as 30 min (Figure 2A). Pretreatment with FIZZ1 caused a reduction in $\mathrm{TNF} \alpha / \mathrm{CHX}$ activation of caspase- 3 at $4 \mathrm{~h}$, and caspase- 8 at 1 and $4 \mathrm{~h}$ of $\mathrm{TNF} \alpha$ treatment (Figure 2A, B). The results were confirmed using a colorimetric assay for caspase-3 (Figure 2C). Thus, inhibition of apoptosis by FIZZ1 was accompanied by reduction in activation of caspases- 3 and caspase-8.

(a)

\begin{tabular}{|c|c|c|c|}
\hline TIME (hours): & 0.5 & 1.0 & 4.0 \\
\hline FIZZ1 & $-\quad+$ & $\begin{array}{ll}- & +\end{array}$ & - \\
\hline TNF $\alpha / C H X$ & $+\quad+$ & + & + \\
\hline Caspase-3 & & & $\infty-\infty$ \\
\hline Caspase-8 & $-\infty$ & -10 & $\infty-\infty$ \\
\hline GAPDH & & & \\
\hline
\end{tabular}

(c)

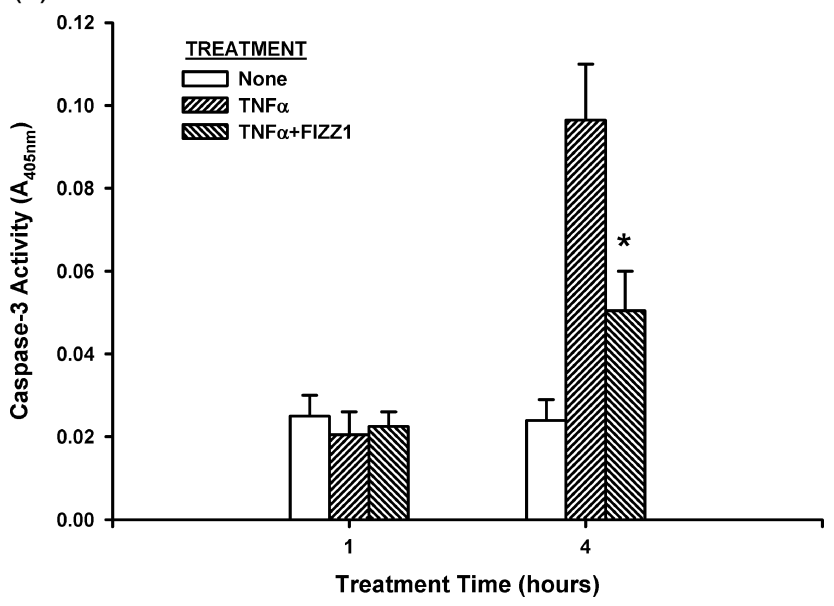

\section{FIZZI effects on Akt and ERK}

To further determine the intracellular mechanism involved in the antiapoptotic effect of FIZZ1, we analysed the effect of FIZZ1 on signalling pathways that are known to control cell survival, such as the PI-3 kinase-Akt and ERK pathways. Western blotting analysis for Akt and ERK and their respective phosphorylated species (pAkt and pERK, respectively) showed that FIZZ1 alone did not activate Akt, but rapidly activated ERK (Figure 3A).

In contrast to FIZZ1 treatment alone, combined treatment with $\mathrm{TNF} \alpha / \mathrm{CHX}$ caused a noticeable increase in pAkt at 10 and $60 \mathrm{~min}$ of treatment (Figure 3B), indicative of Akt activation under this condition. ERK activation was enhanced by the combined treatment after 10 min compared to FIZZ1 treatment alone, but also appeared diminished by $60 \mathrm{~min}$. The levels of total ERK and Akt were not noticeably affected by any of the treatments. Pretreatment with

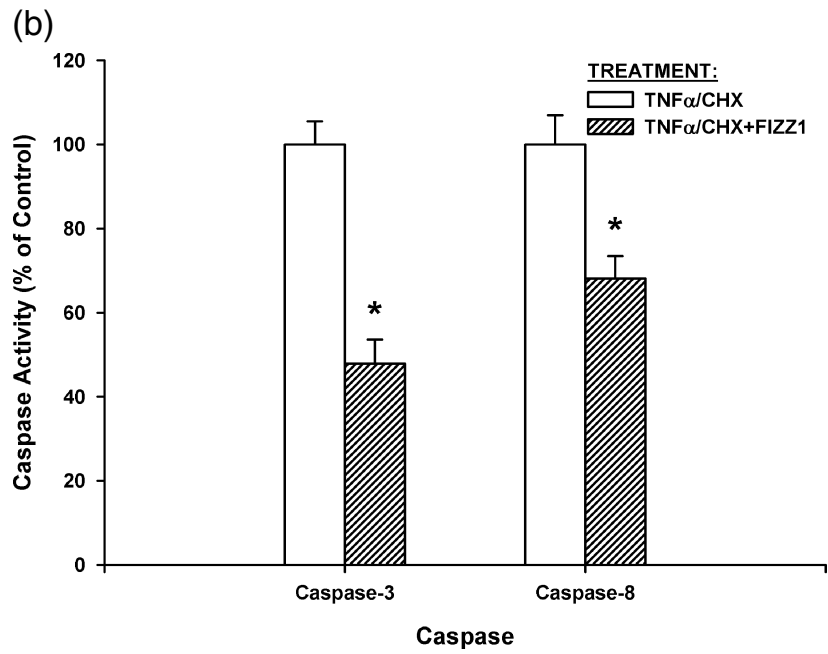

Figure 2. FIZZI effect on TNF $\alpha / \mathrm{CHX}$-induced caspase-activation. Apoptosis of MLF was induced by $5 \mathrm{ng} / \mathrm{ml} \mathrm{TNF} \alpha / 500 \mathrm{ng} / \mathrm{ml}$ $\mathrm{CHX}$ treatment for $4 \mathrm{~h}$ after cell culture in the absence or presence of FIZZI for $24 \mathrm{~h}$. Caspase-3 and -8 activities were analysed by Western blot (A, B) and colorimetric assay (C, caspase-3 only), as described in Methods. Densitometric analysis of caspase-3 and -8 expression levels normalized to GAPDH at $4 \mathrm{~h}$ is shown in (B). Results are shown as percentages of the respective control (untreated) values. The results of colorimetric assay are expressed as mean \pm SE of triplicate measurements. Asterisks in (B, C) indicate that the mean value for the TNF $\alpha+F I Z Z I$ group was significantly less $(p<0.05)$ than that for cells treated with $\mathrm{TNF} \alpha / \mathrm{CHX}$ ('TNF $\alpha$ ') only, as assessed by ANOVA 
(a)

\begin{tabular}{|c|c|c|c|c|}
\hline TIME (hours): & 0.08 & 0.5 & 6.0 & 24.0 \\
\hline FIZZ1 & $-\quad+$ & $-\quad+$ & $-\quad+$ & $-\quad+$ \\
\hline P-Akt & $3=$ & - & $=$ & $=-$ \\
\hline Akt & & & & $=-$ \\
\hline P-ERK & - & - & -- & - \\
\hline ERK & -- & - & -- & -- \\
\hline
\end{tabular}

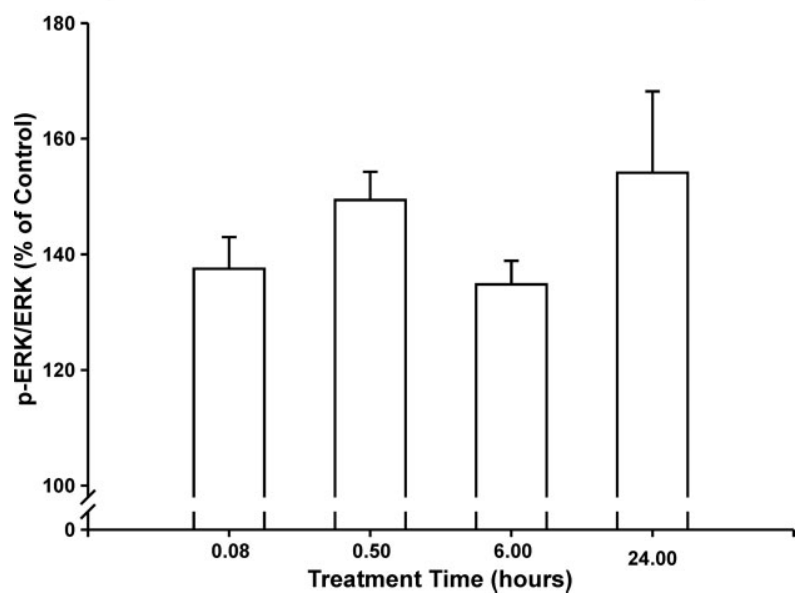

(c)

\begin{tabular}{|c|c|c|c|}
\hline FIZZ1 & $-\quad+$ & + & + \\
\hline PD98059 & - & + & - \\
\hline LY294002 & $-\quad-$ & - & + \\
\hline ERK & $=0$ & & - \\
\hline P-ERK & - & & - \\
\hline
\end{tabular}

(b)

\begin{tabular}{|c|c|c|c|}
\hline TIME (mins): & 10 & 60 & 240 \\
\hline FIZZ1 & $-\quad+$ & $-\quad+$ & $-\quad+$ \\
\hline $\mathrm{TNF} \alpha / \mathrm{CHX}$ & $+\quad+$ & $+\quad+$ & $+\quad+$ \\
\hline Akt & -1 & -0 & -2 \\
\hline P-Akt & -- & -- & $-\cdots$ \\
\hline ERK & $\Longrightarrow$ & $=$ & $=$ \\
\hline P-ERK & $-=$ & - & $=$ \\
\hline
\end{tabular}

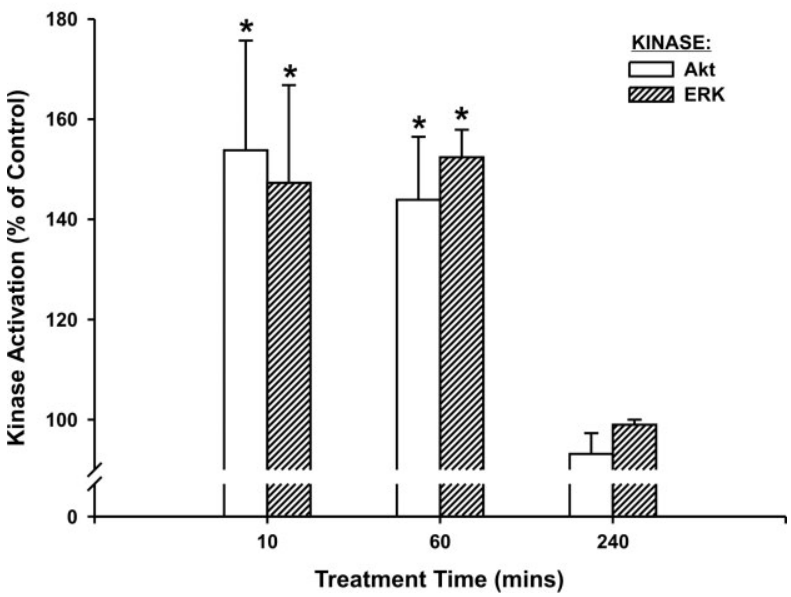

Figure 3. Effects on ERK and Akt activation. Serum-deprived MLFs were treated with FIZZI only (A), FIZZI $+\mathrm{TNF} \alpha / \mathrm{CHX}$ $(B)$ or with FIZZI + the indicated kinase inhibitor $(C)$. They were then analysed for the indicated signalling proteins and their phosphorylated forms by western blotting. Representative western blots are shown, which were repeated twice with comparable results. The lower panel in (A) shows the result of densitometric analysis of phosphorylated ERK (P-ERK) level normalized to total ERK, and expressed as a percentage of the control (untreated cells) mean value. In (B) the results of similar densitometric analysis of P-Akt and P-ERK levels (normalized to their respective total values) as a function of time are shown in the lower panel. Asterisks indicate significant increase $(p<0.05)$ above untreated control mean values. FIZZI activated ERK (A), but required combined treatment with TNF $\alpha / \mathrm{CHX}$ to activate Akt (B). The MEK inhibitor, PD98059, inhibited FIZZI-induced ERK activation (C, bottom row)

the MEK/ERK inhibitor PD98059 reduced the FIZZ1induced ERK phosphorylation, but was not affected by the PI3 kinase inhibitor LY 294002 (Figure 3C). Thus, inhibition of TNF $\alpha$-induced apoptosis by FIZZ1 was accompanied by activation of both Akt and ERK signalling, although FIZZ1 alone had no significant effect on Akt signalling.

The effects of these kinase inhibitors on apoptosis were then examined. Addition of the MEK inhibitor PD98059 alone caused enhanced activation of caspase- 3 relative to that caused by $\mathrm{TNF} \alpha / \mathrm{CHX}$ alone, while LY294002 did not have a noticeable effect (Figure 4). Furthermore, PD98059 reversed the inhibitory effect of FIZZ1 on $\mathrm{TNF} \alpha / \mathrm{CHX}$-induced activation of caspase- 3 and -8. The PI3 kinase inhibitor did not have a detectable effect on the activation of caspase-8. Moreover, relative to cells transfected with the expression transfection vector only (PCIS2), transfection with a dominant negative Akt construct (Akt-K179A) did not significantly affect $\mathrm{TNF} \alpha / \mathrm{CHX}$-induced caspase- 8 activity, neither did it affect inhibition of this induced activity by FIZZ1 (Figure 5). Thus, only the ERK pathway appeared to be significantly involved in mediating the FIZZ1 signal. To further confirm the importance of ERK in mediating the FIZZ1 antiapoptotic effect, the effect of PD98059 on annexin V staining was evaluated. The results showed that the FIZZ1-induced reduction 
(a)

\begin{tabular}{|r|ll|ll|ll|}
\hline TNF $\alpha / C H X$ & + & + & + & + & + & + \\
\hline FIZZ1 & - & + & - & + & - & + \\
\hline PD98059 & - & - & + & + & - & - \\
\hline LY294002 & - & - & - & - & + & + \\
\hline Caspase-3 & - & - & - & & - & \\
\hline Caspase-8 & - & - & - & & & \\
\hline GAPDH & & & & & & \\
\hline
\end{tabular}

(b)

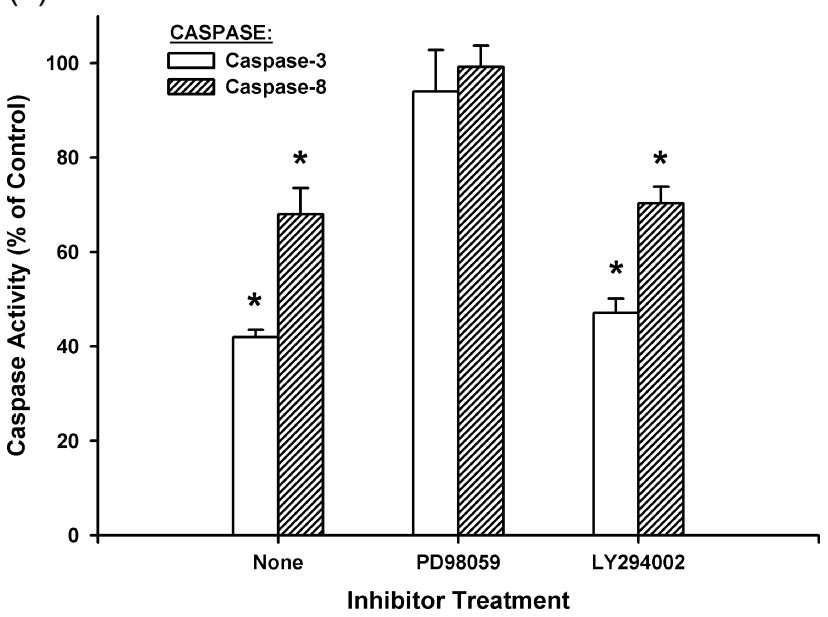

Figure 4. Effects of kinase inhibition on caspase activity. The cells were treated as indicated and the protein extracts were then analysed by western blotting for caspase- 3 and -8 (A). A representative blot is shown, which was repeated twice with comparable results. The results of quantification by densitometry and after normalization to GAPDH are shown in (B) as a percentage of the mean value of cells treated with $\mathrm{TNF} \alpha / \mathrm{CHX}$ only (i.e. without $\mathrm{FIZZI}$ ). Asterisks indicate significant difference $(p<0.05)$ from mean values of $\mathrm{TNF} \alpha / \mathrm{CHX}$-treated cells. FIZZI suppression of $\mathrm{TNF} \alpha / \mathrm{CHX}$-induced caspase- 3 and -8 activation was abolished by the MEK inhibitor, PD98059, but not by LY294002

in apoptosis was completely reversed by treatment with PD98059 (Figure 6). These data indicated that the FIZZ1 antiapoptotic activity was mediated by the ERK MAP kinase pathway, as manifested by inhibition of caspase- 3 and -8 activation, as well as by annexin $\mathrm{V}$ immunostaining.

\section{Discussion}

This report demonstrates that FIZZ1 has antiapoptotic activity in primary cultured MLFs and that this effect is associated with activation of ERK and inhibition of caspase- 3 and -8. Since FIZZ1 also promotes myofibroblast differentiation [4], this would contribute to the

\begin{tabular}{|r|ccc|ccc|}
\hline TRANSFECTANT: & \multicolumn{2}{|c|}{ PCIS2 } & \multicolumn{2}{|c|}{ Akt-K179A } \\
\hline TNF $\alpha / C H X$ & - & + & + & - & + & + \\
\hline FIZZ1 & - & - & + & - & - & + \\
\hline Caspase-8 & - & - & - & - & - & - \\
\hline GAPDH & & & & & & \\
\hline & & & & & & \\
\hline
\end{tabular}

Figure 5. Effects of Akt mutant transfection on caspase activity. MLFs were transfected with the dominant negative Akt construct (Akt-KI79A) or expression vector (PCIS2) only. Cells were treated as indicated and the protein extracts were then analysed by western blotting for caspase- 8 activity. FIZZI suppression of $\mathrm{TNF} \alpha / \mathrm{CHX}$-induced caspase- 8 activation was not abolished by the dominant negative Akt construct

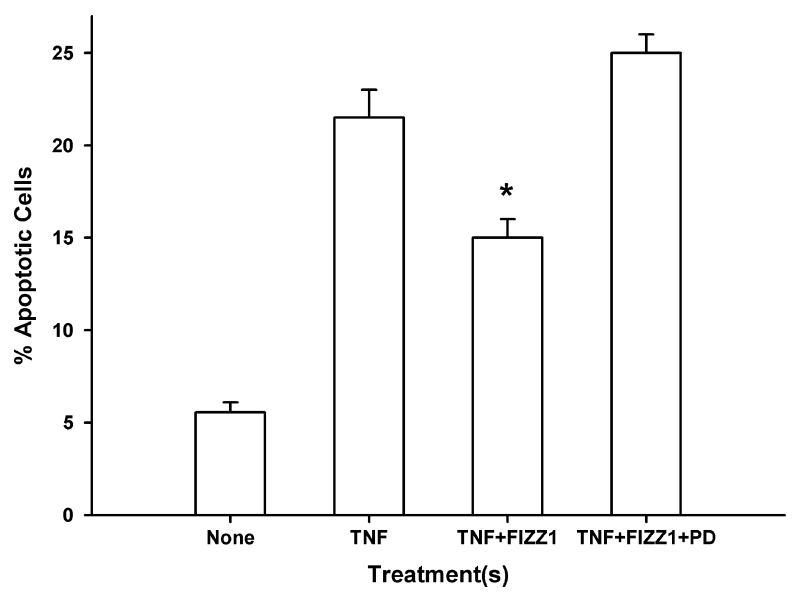

Figure 6. Effects of kinase inhibition on apoptosis. Cells were treated as indicated (TNF, TNF $\alpha / \mathrm{CHX}$; TNF + FIZZI, $\mathrm{TNF} \alpha / \mathrm{CHX}$ and $\mathrm{FIZZI} ; \mathrm{TNF}+\mathrm{FIZZI}+\mathrm{PD}, \mathrm{TNF} \alpha / \mathrm{CHX}$, FIZZI and PD98059) and apoptosis was detected by FACS analysis, as described in Methods. Results are shown as mean \pm $\mathrm{SE}(n=3)$. An asterisk indicates significant difference $(p<0.05)$ from the mean value of the 'TNF' group. PD98059 reversed the inhibitory effect of $\mathrm{FIZZI}$ on TNF $\alpha / \mathrm{CHX}$-induced apoptosis

enhanced survival and persistence of the myofibroblast. In response to pulmonary injury with accompanying inflammation, myofibroblasts play a key role as a major cellular source of extracellular matrix deposition, a hallmark of pulmonary fibrosis. In normal wound healing, the de novo genesis of myofibroblasts peaks at the height of active fibrosis with elaboration of matrix components and subsequently gradually declines as the healing process is successfully completed. This decline in myofibroblast numbers is thought to be due to their apoptosis, although the trigger or inducer of apoptosis remains uncertain $[12,13]$. In contrast, these cells persist and can be found in various stages of human pulmonary fibrosis where the disease is progressive. Therefore, the altered regulation of the number of myofibroblasts may be a key factor in progressive pulmonary fibrosis [14-16].

Apoptosis is a physiological process leading to elimination of unwanted cells within living tissues [17]. Studies of wound healing suggest that myofibroblast 
disappearance occurs via apoptosis [12]. The mechanisms involved in physiological or pathological regulation of myofibroblast apoptosis are unknown, and growth factors, cytokines, extracellular matrix interaction or death receptor ligands appear to be potential modulators $[13,18]$. A role for cytokines as modulators of apoptosis susceptibility has also been proposed, but data on the effect of pro- or antifibrotic cytokines on fibroblast apoptosis are scanty. Transforming growth factor- $\beta$ (TGF $\beta$ ), one of the most potent fibrogenic cytokines, promotes myofibroblast survival by preventing the myofibroblast from undergoing apoptosis [19]. In contrast to the effect of $\operatorname{TGF} \beta$, interleukin- $1 \beta$ (via generation of $\mathrm{NO}$ ) and the CD95-receptor-CD95ligand system (Fas/FasL) are reported to induce apoptosis $[20,21]$. The present study revealed that, similar to $\mathrm{TGF} \beta$, FIZZ1 had an antiapoptotic effect on MLF, which was associated with myofibroblast differentiation [4]. The response that we observed is similar to a FIZZ1-induced inhibition of apoptosis noted in lung epithelial cells from lipopolysaccharide-induced apoptosis and in cultured embryonic lung [10,22]. This suggests that the antiapoptotic effect of FIZZ1 may be a generalized activity that is independent of its ability to induce myofibroblast differentiation. This possibility, however, cannot be ruled out from the data presented.

Although there is evidence that FIZZ1 has significant antiapoptotic activity in lung epithelial cells and fibroblasts, the mechanisms involved in the antiapoptotic effect of FIZZ1 are still unknown. The caspases are the ultimate effectors or executioners of programmed cell death. They are synthesized as inactive precursors that are usually converted to the active form by proteolytic cleavage, catalysed by other caspases. TNF $\alpha$ is known to induce apoptosis by modulating Fas-associated death domain-like IL-1-converting enzyme-like inhibitory protein (FLIP), caspase-8, and surface Fas expression [21]. Caspase- 8 activation is capable of inducing apoptosis mediated either directly through the activation of caspase-3, or by the activation of the Bid protein, which results in mitochondrial injury, the release of cytochrome $c$, and activation of caspase-9 and caspase-3. To characterize the mechanism of the antiapoptotic effect of FIZZ1 in MLF, the activation of caspase- 8 and one of its downstream targets, caspase-3, was examined. FIZZ1 was found to be effective at inhibiting caspase- 8 and -3 activation, correlating with its antiapoptotic activity in these cells. These observations suggested that FIZZ1 could prevent apoptosis of MLF by inhibiting the activation of these caspases, which could then contribute to the progression of pulmonary fibrosis by promoting myofibroblast survival and persistence.

Cell survival, death and apoptosis are also known to be regulated by certain mitogen-activated protein kinase (MAPK) pathways. MAPKs are cytoplasmic serine/threonine kinases that are activated in response to a various extracellular stimuli, including those that regulate cell proliferation, differentiation, cell survival, development and inflammation. ERK is a member of the MAPKs, and activation of the ERK pathway suppresses apoptosis induced by growth factor deprivation, matrix detachment signals and cytotoxic drugs [23-26]. Another major intracellular signalling pathway that is responsible for promoting cell survival is the PI3-kinase-Akt signalling pathway. Activation of PI3-kinase is thought to inhibit apoptosis by promoting translocation of Akt to the plasma membrane, where Akt can be phosphorylated at Thr308 and Ser473 by the kinases PDK-1 and PDK-2, respectively [27,28]. Phosphorylated Akt functions as a survival signal by inhibiting the activation of pro-apoptotic signals [29]. Neither the receptor nor the downstream signalling pathway for FIZZ1 has been identified with respect to its antiapoptotic activity. In rat pulmonary vascular smooth muscle cells, PI3-kinase and Akt are involved in FIZZ1 downstream signalling related to their proliferation. A recent study suggests that FIZZ1 induces vascular adhesion molecule-1 (VCAM-1) expression via the $\mathrm{PI} 3 \mathrm{~K}-\mathrm{Akt}-\mathrm{NF} \kappa \mathrm{B}$ signalling pathway in mouse endothelial and lung epithelial cells [30]. FIZZ2, another FIZZ family member, activates ERK and p38 in hepatocytes that is related to insulin resistance. [31]. However, there is so far no report that ERK participates in FIZZ1 downstream signalling. ERK1/2 was was activated upon treatment with FIZZ1, and this activation was suppressed by an inhibitor of this pathway, the MEK/ERK inhibitor PD98059. Furthermore, we demonstrated that FIZZ1-induced ERK activation was associated with inhibition of caspase- 3 activation. This result is consistent with previous reports that activation of ERK is associated with inhibition of caspase-3 activation. ERK phosphorylates caspase9 on Thr125 and it inhibits caspase- 9 processing and caspase-3 activation [32,33].

In contrast to ERK, Akt was not activated by FIZZ1, except when combined with $\mathrm{TNF} \alpha / \mathrm{CHX}$ treatment. Thus, while the importance of ERK in FIZZ1-induced antiapoptotic activity was clearly suggested, the role of Akt was less clear and may involve more complex mechanisms associated with the additional response to $\mathrm{TNF} \alpha / \mathrm{CHX}$ treatment. Moreover, the importance of ERK was confirmed by the ability of PD98059 to abrogate the antiapoptotic effect of FIZZ1, including its effects on caspase activation, while the significance of Akt was not apparent, since LY294002 failed to affect the FIZZ1-induced inhibition of caspase activation. Taken together, these data indicate that the ERK pathway is significantly involved in the antiapoptotic effect of FIZZ1. Whether this effect is dependent on the myofibroblast differentiation also promoted by FIZZ1 [4] remains to be determined. In any case, the data presented in this study suggest that FIZZ1 can contribute to the pathogenesis of progressive pulmonary fibrosis by induction of myofibroblast persistence via antiapoptotic effects.

\section{Acknowledgements}

We thank Dr Victor Thannickal (Department of Internal Medicine, University of Michigan) for the generous gift of the 
dominant negative Akt construct (Akt-K179A) and expression vector control (PCIS2). This work was supported by grants HL28737, HL31963, HL52285 and HL77297 from the National Institutes of Health, and an award to S.H.P. from the Sandler Program for Asthma Research.

\section{References}

1. Holcomb IN, Kabakoff RC, Chan B, Baker TW, Gurney A, Henzel W, et al. FIZZ1, a novel cysteine-rich secreted protein associated with pulmonary inflammation, defines a new gene family. EMBO J 2000;19:4046-4055.

2. Steppan CM, Brown EJ, Wright CM, Bhat $S$, Banerjee RR, Dai CY, et al. A family of tissue-specific resistin-like molecules. Proc Natl Acad Sci USA 2001;98:502-506.

3. Gerstmayer B, Kusters D, Gebel S, Muller T, Van Miert E, Hofmann $\mathrm{K}$, et al. Identification of RELM gamma, a novel resistin-like molecule with a distinct expression pattern. Genomics 2003;81:588-595.

4. Liu T, Dhanasekaran SM, Jin H, Hu B, Tomlins SA, Chinnaiyan AM, et al. FIZZ1 stimulation of myofibroblast differentiation. Am J Pathol 2004;164:1315-1326.

5. Liu T, Jin H, Ullenbruch M, Hu B, Hashimoto N, Moore B, et al. Regulation of found in inflammatory zone 1 expression in bleomycin-induced lung fibrosis: role of IL-4/IL-13 and mediation via STAT-6. J Immunol 2004;173:3425-3431.

6. Teng X, Li D, Champion HC, Johns RA. FIZZ1/RELM $\alpha$, a novel hypoxia-induced mitogenic factor in lung with vasoconstrictive and angiogenic properties. Circ Res 2003;92:1065-1067.

7. Yamaji-Kegan K, Su Q, Angelini DJ, Champion HC, Johns RA. Hypoxia-induced mitogenic factor has pro-angiogenic and proinflammatory effects in the lung via VEGF and VEGF receptor-2. Am J Physiol Lung Cell Mol Physiol 2006;(in press).

8. Nair MG, Cochrane DW, Allen JE. Macrophages in chronic type 2 inflammation have a novel phenotype characterized by the abundant expression of Ym1 and Fizz1 that can be partly replicated in vitro. Immunol Lett 2003;85:173-180.

9. Raes G, De Baetselier P, Noel W, Beschin A, Brombacher F, Hassanzadeh Gh G. Differential expression of FIZZ1 and Ym1 in alternatively versus classically activated macrophages. J Leukoc Biol 2002;71:597-602.

10. Wagner KF, Hellberg AK, Balenger S, Depping R, Dodd-O J, Johns RA, et al. Hypoxia-induced mitogenic factor has antiapoptotic action and is upregulated in the developing lung: coexpression with hypoxia-inducible factor-2alpha. Am J Respir Cell Mol Biol 2004;31:276-282.

11. Phan SH, Varani J, Smith D. Rat lung fibroblast collagen metabolism in bleomycin-induced pulmonary fibrosis. J Clin Invest 1985;76:241-247.

12. Desmouliere A, Redard M, Darby I, Gabbiani G. Apoptosis mediates the decrease in cellularity during the transition between granulation tissue and scar. Am J Pathol 1995;146:56-66.

13. Jelaska A, Korn JH. Role of apoptosis and transforming growth factor $\beta 1$ in fibroblast selection and activation in systemic sclerosis. Arthritis Rheum 2000;43:2230-2239.

14. Darby I, Skalli O, Gabbiani G. Alpha-smooth muscle actin is transiently expressed by myofibroblasts during experimental wound healing. Lab Invest 1990;63:21-29.

15. Majno G, Gabbiani G, Hirschel BJ, Ryan GB, Statkov PR. Contraction of granulation tissue in vitro: similarity to smooth muscle. Science 1971;173:548-550.
16. Kuhn C, McDonald JA. The roles of the myofibroblast in idiopathic pulmonary fibrosis. Ultrastructural and immunohistochemical features of sites of active extracellular matrix synthesis. Am J Pathol 1991;138:1257-1265.

17. Kerr JF, Wyllie AH, Currie AR. Apoptosis: a basic biological phenomenon with wide-ranging implications in tissue kinetics. $\mathrm{Br}$ J Cancer 1972;26:239-257.

18. Fluck J, Querfeld C, Cremer A, Niland S, Krieg T, Sollberg S. Normal human primary fibroblasts undergo apoptosis in three-dimensional contractile collagen gels. J Invest Dermatol 1998;110:153-157.

19. Zhang HY, Phan SH. Inhibition of myofibroblast apoptosis by transforming growth factor beta(1). Am J Respir Cell Mol Biol 1999;21:658-665.

20. Zhang HY, Gharaee-Kermani M, Phan SH. Regulation of lung fibroblast alpha-smooth muscle actin expression, contractile phenotype, and apoptosis by $\mathrm{IL}-1 \beta$. J Immunol 1997;158:1392-1399.

21. Santiago B, Galindo M, Palao G, Pablos JL. Intracellular regulation of Fas-induced apoptosis in human fibroblasts by extracellular factors and cycloheximide. $J$ Immunol 2004;172:560-566.

22. Tong Q, Zheng L, Kang Q, Dodd-O J, Langer J, Li B, et al. Upregulation of hypoxia-induced mitogenic factor in bacterial lipopolysaccharide-induced acute lung injury. FEBS Lett 2006;580:2207-2215.

23. Xia Z, Dickens M, Raingeaud J, Davis RJ, Greenberg ME. Opposing effects of ERK and JNK-p38 MAP kinases on apoptosis. Science 1995;270:1326-1331.

24. Le Gall M, Chambard JC, Breittmayer JP, Grall D, Pouyssegur J, Van Obberghen-Schilling E. The p42/p44 MAP kinase pathway prevents apoptosis induced by anchorage and serum removal. Mol Biol Cell 2000;11:1103-1112.

25. Rytomaa M, Lehmann K, Downward J. Matrix detachment induces caspase-dependent cytochrome $c$ release from mitochondria: inhibition by PKB/Akt but not Raf signalling. Oncogene 2000;19:4461-4468.

26. MacKeigan JP, Collins TS, Ting JP. MEK inhibition enhances paclitaxel-induced tumor apoptosis. $J$ Biol Chem 2000;275:38953-38956.

27. Downward J. Mechanisms and consequences of activation of protein kinase B/Akt. Curr Opin Cell Biol 1998;10:262-267.

28. Roymans D, Slegers H. Phosphatidylinositol 3-kinases in tumor progression. Eur J Biochem 2001;268:487-498.

29. Datta SR, Brunet A, Greenberg ME. Cellular survival: a play in three Akts. Genes Dev 1999;13:2905-2927.

30. Tong Q, Zheng L, Lin L, Li B, Wang D, Li D. Hypoxiainduced mitogenic factor promotes vascular adhesion molecule-1 expression via the PI-3K/Akt-NF- $\kappa$ B signalling pathway. Am $J$ Respir Cell Mol Biol 2006;35:444-456.

31. Kushiyama A, Shojima N, Ogihara T, Inukai K, Sakoda H, Fujishiro M, et al. Resistin-like molecule beta activates MAPKs, suppresses insulin signalling in hepatocytes, and induces diabetes, hyperlipidemia, and fatty liver in transgenic mice on a high fat diet. J Biol Chem. 2005;23:42016-42025.

32. Allan LA, Morrice N, Brady S, Magee G, Pathak S, Clarke PR. Inhibition of caspase- 9 through phosphorylation at Thr 125 by ERK MAPK. Nat Cell Biol 2003;5:647-654.

33. Tashker JS, Olson M, Kornbluth S. Post-cytochrome $c$ protection from apoptosis conferred by a MAPK pathway in Xenopus egg extracts. Mol Biol Cell 2002;13:393-401. 